\title{
Local and network behavior of bistable vibrational energy harvesters considering periodic and quasiperiodic excitations
}

Cite as: Chaos 31, 063111 (2021); https://doi.org/10.1063/5.0054459

Submitted: 19 April 2021 • Accepted: 24 May 2021 • Published Online: 07 June 2021

(D) Karthikeyan Rajagopal, Arthanari Ramesh, Irene Moroz, et al.

\section{COLLECTIONS}

Paper published as part of the special topic on In Memory of Vadim S. Anishchenko: Statistical Physics and Nonlinear Dynamics of Complex Systems
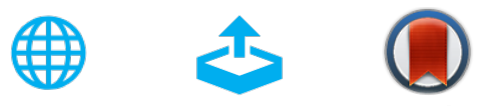

Export Citation

\section{ARTICLES YOU MAY BE INTERESTED IN}

Extreme synchronization events in a Kuramoto model: The interplay between resource constraints and explosive transitions

Chaos: An Interdisciplinary Journal of Nonlinear Science 31, 063103 (2021); https:// doi.org/10.1063/5.0055156

Noise induced suppression of spiral waves in a hybrid FitzHugh-Nagumo neuron with discontinuous resetting

Chaos: An Interdisciplinary Journal of Nonlinear Science 31, 073117 (2021); https:// doi.org/10.1063/5.0059175

Sparse optimization of mutual synchronization in collectively oscillating networks Chaos: An Interdisciplinary Journal of Nonlinear Science 31, 063113 (2021); https:// doi.org/10.1063/5.0049091

\section{Scilight}

Summaries of the latest breakthroughs in the physical sciences 


\title{
Local and network behavior of bistable vibrational energy harvesters considering periodic and quasiperiodic excitations
}

Cite as: Chaos 31, 063111 (2021); doi: 10.1063/5.0054459

Submitted: 19 April 2021 . Accepted: 24 May 2021 .

Published Online: 7 June 2021

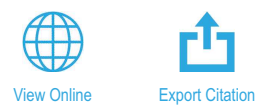

Karthikeyan Rajagopal, , ,a) (D) Arthanari Ramesh,, 2, b) Irene Moroz,, ${ }^{3, c)}$ Prakash Duraisamy, ,d) (D) and Anitha Karthikeyan ${ }^{4, e)}$

\begin{abstract}
AFFILIATIONS
${ }^{1}$ Center for Nonlinear Systems, Chennai Institute of Technology, Chennai 600069, India

${ }^{2}$ Center for Materials Research, Chennai Institute of Technology, Chennai 600069, India

${ }^{3}$ Mathematical Institute, University of Oxford, Andrew Wiles Building, Oxford OX2 6CG, United Kingdom

${ }^{4}$ Department of Electronics and Communication Engineering, Prathyusha Engineering College, Thiruvallur, Tamil Nadu 602025, India
\end{abstract}

Note: This paper is part of the Focus Issue, In Memory of Vadim S. Anishchenko: Statistical Physics and Nonlinear Dynamics of Complex Systems.

a) Author to whom correspondence should be addressed: karthikeyan.rajagopal@citchennai.net

b) ramesha@citchennai.net
c)irene.Moroz@maths.ox.ac.uk
d) prakash.duraisamy@citchennai.net
e)mrs.anithakarthikeyan@gmail.com

\begin{abstract}
Vibrational energy harvesters can exhibit complex nonlinear behavior when exposed to external excitations. Depending on the number of stable equilibriums, the energy harvesters are defined and analyzed. In this work, we focus on the bistable energy harvester with two energy wells. Though there have been earlier discussions on such harvesters, all these works focus on periodic excitations. Hence, we are focusing our analysis on both periodic and quasiperiodic forced bistable energy harvesters. Various dynamical properties are explored, and the bifurcation plots of the periodically excited harvester show coexisting hidden attractors. To investigate the collective behavior of the harvesters, we mathematically constructed a two-dimensional lattice array of the harvesters. A non-local coupling is considered, and we could show the emergence of chimeras in the network. As discussed in the literature, energy harvesters are efficient if the chaotic regimes can be suppressed and hence we focus our discussion toward synchronizing the nodes in the network when they are not in their chaotic regimes. We could successfully define the conditions to achieve complete synchronization in both periodic and quasiperiodically excited harvesters.
\end{abstract}

Published under an exclusive license by AIP Publishing. https://doi.org/10.1063/5.0054459

Vibration energy harvesters (VEHs) exhibit rich dynamical properties when excited with periodic excitations as shown in the literature. Though such discussions are abundant, there is no literature discussing the effect of quasiperiodic excitations. Also, we have discussed multistability and coexisting attractors in a bistable energy harvester and have shown that a quasiperiodic excited energy harvester does not exhibit multistability. Though local dynamics of the VEHs are significant, it is its network behavior which is very important to investigate the synchronization behavior as a periodic VEH completely synchronized will be efficient against a chaotic VEH exhibiting chimera states.

\section{INTRODUCTION}

Wireless sensors and other low powered health monitoring devices have been widely used in industrial, medical, military, 
engineering, and environmental health monitoring areas. ${ }^{1-3}$ These devices need a continuous power supply with the least replacement cost and long-lifetime service. Their power supply requirements are not fully addressed using the traditional batteries. ${ }^{4}$ Vibration energy is readily available in the environment in the form of wind, ocean waves, human motion, and mechanical vibration. ${ }^{5}$ Among these, the mechanical energy is one of the most suitable one for structural health monitoring due to its availability. ${ }^{4}$ As a result, energy harvesting from mechanical vibration is a promising means to replace conventional power sources.

Linear resonant energy harvesters have been used to exploit ambient vibration energy based on the linear resonant vibration principle. They are effective only if the excitation power is concentrated in a narrow band of frequency, i.e., stationary excitation. But when the excitation frequency does not match the resonant frequency, the harvesting efficiency reduced severely. Many researchers tried to solve this issue by expanding the bandwidth of frequency using techniques such as frequency upconversion, resonance tuning, and deliberate introduction of nonlinearity. ${ }^{6-12}$ Among these, the inclusion of nonlinearity to harvesters attracted much attention. Monostable and bistable harvesters found their significance due to various advantages under different excitation conditions and initial conditions. Monostable harvesters hold a simple configuration and have one stable equilibrium state. Compared to linear systems, they are able to widen the frequency response range. ${ }^{8}$ But their performance drops when they are subjected to real-world scenarios such as random excitation and have a similar output to linear harvesters when they are excited by white noise vibrations. ${ }^{13}$ As an improvement to the drawback of monostable harvesters, researchers have focused on multistable harvesters. The bistable harvester has been explored widely. ${ }^{14-17}$ It has two potential wells with a barrier in between. If the excitation amplitude is high enough, the system will jump between the stable wells creating an interwell oscillation with high amplitude. The shape of the wells influences the response of the system. When the wells are shallow, the bandwidth of the harvester increases; however, the amplitude of the response is reduced. Compared to monostable harvesters, bistable shows improvement in harvesting, when the amplitude of the excitation is large enough to trigger an interwell oscillation. ${ }^{18,19}$

In addition to that, there is only minimal literature found for studying the network behaviors of energy harvesters but practically, they should work in a network to obtain a significant output. The coupling effect plays an important role in network dynamics and shows significant results in output energy. If the network of harvesters is not properly tuned, there is chance of energy dissipation in nodes. Hence, there is a huge demand in finding proper coupling strength and periodic frequency. The study of an interesting phenomenon on synchronization ${ }^{20,21}$ and incoherent oscillations of a horde non-locally coupled oscillators ${ }^{22}$ is pronounced as "chimera states." The dynamics of chimera states and different topologies to handle chimera has been investigated vigorously in recent literature. ${ }^{23-25}$ The existence of chimera states and their control are studied with experimental evidence in some literature. ${ }^{26-31}$ Hence, considering undeniable mutual effects of chimera states in dynamics of network and spatiotemporal nature, the intriguing characteristics of the system can be analyzed and it becomes a fruitful test ground for energy efficiency also.
Motivated from the above discussion, in this paper, we formulated a Bistable Energy Harvester (BEH) supplied with higher order nonlinearity under quasi-periodic excitation. Stability analysis is carried out and presented in Sec. III. Bifurcation plots and the corresponding Lyapunov spectrum are derived for different scenarios in Sec. IV. The major contribution of this work lies on the network dynamics of $\mathrm{BEH}$ under periodic and quasi-periodic excitations, the simulations are portrayed and interpreted in Sec. V. Finally, we provided concluding remarks and highlighted the significance of the present study.

\section{MATHEMATICAL MODELING}

In Fig. 1, the configuration of a nonlinear energy harvester is shown. The configuration of a bistable energy harvester consists of a stainless-steel substrate with two lead zirconate titanate (PZT) piezoelectric layers positioned near the base and two tip magnets. There are two external magnets located with the required distance and angle.

The equation of motion for the $\mathrm{BEH}$ configuration presented in Fig. 1 is derived as follows:

$$
\begin{array}{r}
m \ddot{r}(t)+c \dot{r}(t)+F_{h}-\theta V(t)=\Phi(t), \\
C_{p} \dot{V}(t)+\frac{V(t)}{R}+\theta \dot{r}(t)=0,
\end{array}
$$

where $m$ and $c$ are the equivalent mass and damping, $C_{p}$ refers to the equivalent capacitance, $\theta$ represents the electromechanical coupling coefficient of the piezoelectric material, $R$ denotes the load resistance, $V$ denotes the voltage, $r$ is the displacement, and $\Phi(t)$ refers to the external excitation.

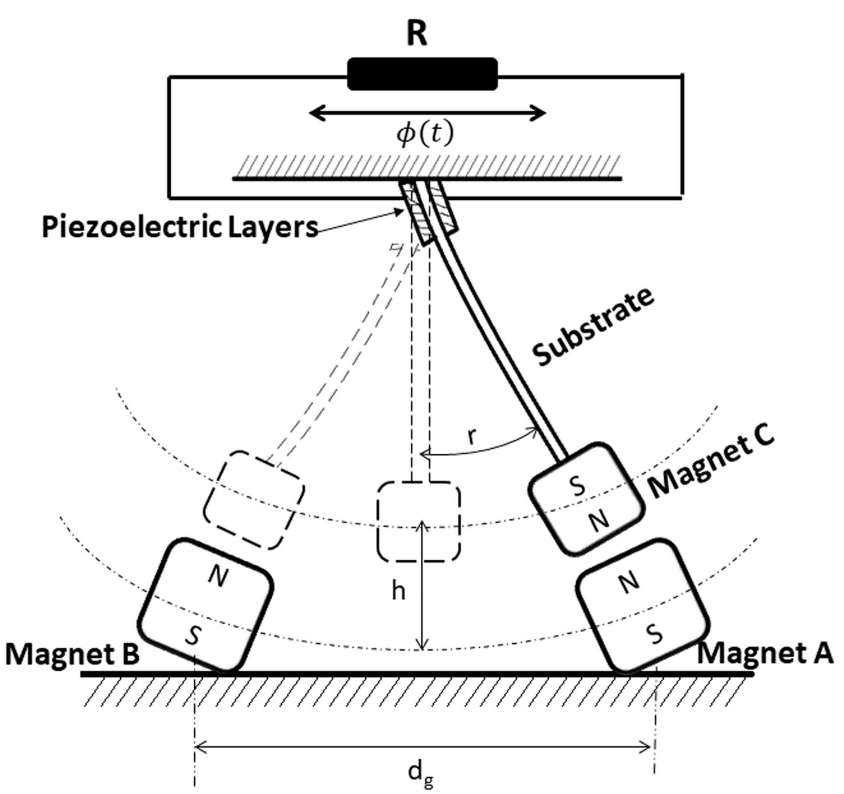

FIG. 1. Configuration of bistable energy harvester (BEH). 
For brevity, the equations of motion can be further nondimensionalized by using the following terms:

$$
x=\frac{r}{l_{c}}, \quad t=t \omega_{n}, \quad V=\frac{C_{p}}{\theta l_{c}} V,
$$

where $l_{c}$ is a length scale introduced to nondimensionalize the displacement and $\omega_{n}=\sqrt{\frac{k}{m}}$ is the natural frequency of the harvester. With these transformations, the nondimensional model can be expressed as

$$
\begin{gathered}
\ddot{x}+2 \xi \dot{x}+F_{h}-\kappa^{2} V=\Phi(t), \\
\dot{V}+\alpha V+\dot{x}=0,
\end{gathered}
$$

where

$$
\xi=\frac{c}{2 \sqrt{k_{1} m}}, \delta=\frac{k_{3} l_{c}^{2}}{k_{1}}, \kappa=\frac{\theta^{2}}{k_{1} C_{c}}, \alpha=\frac{1}{\omega_{n} C_{p} R} .
$$

Here, $x$ represents displacement, $\omega_{n}$ is the natural frequency, and $\xi$ refers to the damping ratio. In this paper, we introduced a quartic nonlinearity term as restoring force, and the expression is as follows:

$$
F_{h}=-x+\beta x^{2}+\delta x^{3}+\gamma x^{4}
$$

Most of the analyses done on the existing models are with periodic excitation. Literature on experimental studies of BEH show that quasi-periodic excitation affects the performance significantly; hence, we are introducing quasi-periodic excitation. Generally, quasi-periodic excitation will induce a strange nonchaotic attractor (SNCA) and results in multistability. Hence, in this paper, we considered the system is supplied with quasi-periodic excitation and denoted as $\Phi(t)=A_{1}\left[\sin \left(\omega_{1} t\right)+A_{2} \sin \left(\omega_{2} t\right)\right]$.

The state space equation of the system can be written as

$$
\begin{gathered}
\dot{x}=y, \\
\dot{y}=A_{1}\left[\sin \left(\omega_{1} t\right)+A_{2} \sin \left(\omega_{2} t\right)\right]-2 \xi y+x-\delta x^{3} \\
-\beta x^{2}-\gamma x^{4}+\kappa z, \\
\dot{z}=-y-\alpha z .
\end{gathered}
$$

\section{EQUILIBRIUM POINTS AND STABILITY ANALYSIS}

$F_{h}(x)$ is the nonlinear restoring force, while $\Phi(t)$ is the external excitation: Periodic if $A_{2}=0$ and quasi-periodic if $A_{2} \neq 0$. We take the parameter values in Eq. (5) to be $A_{1}=0.5, \omega_{1}=1, \omega_{2}=\frac{\sqrt{5}-1}{2}$, $A_{2}=1, \quad \xi=0.0933, \delta=0.5495, \quad \beta=0.1, \gamma=0.1, \alpha=0.4065$, $\kappa=0.00185$.

Stanton et al..$^{32}$ performed a Melnikov analysis on a simplified bistable harvester by considering perturbations from a Hamiltonian limit. We follow part of their analysis here.

We rewrite (5) as a perturbed Hamiltonian system,

$$
\left[\begin{array}{c}
\dot{x} \\
\dot{y} \\
\dot{z}
\end{array}\right]=\left[\begin{array}{c}
y \\
F_{h}(x) \\
0
\end{array}\right]-\varepsilon\left[\begin{array}{c}
0 \\
-2 \xi y+\kappa z+f(t) \\
-y-\alpha z
\end{array}\right],
$$

where we have introduced a small parameter $\varepsilon$ to represent the nonHamiltonian terms. When $\varepsilon=0$, Eq. (6) becomes

$$
\begin{gathered}
\dot{x}=y, \\
\dot{y}=x-\beta x^{2}-\delta x^{3}-\gamma x^{4},
\end{gathered}
$$

which leads to the Hamiltonian

$$
E(t)=\frac{1}{2} y^{2}+V(x) .
$$

With the potential energy function $V(x)$

$$
V(x)=-\frac{1}{2} x^{2}+\frac{\beta}{3} x^{3}+\frac{\delta}{4} x^{4}+\frac{\gamma}{5} x^{5} .
$$

The fixed points of (7) satisfy $(x, y)=(0,0), y=0$ and the cubic roots of $1-\beta x-\delta x^{2}-\gamma x^{3}$. The trivial fixed point $(0,0)$ is a saddle point. Values for the parameters, we find the remaining fixed points to be $x_{1}=-4.8675$ (another saddle point), $x_{2}=-1.7810$ and $x_{3}=1.1535$ (both centers).

Figure 2 shows the double homoclinc loop in black refers to the simplified model with $\gamma=0$, homoclinic loop (magenta) that passes through the saddle point $(0,0)$, as well as the large homoclinic loop passes through the saddle point $x_{1}=-4.8675$. For the double homoclinic loop, $E(t)=0$, while for the large homoclinic loop, $E(t)=6.781$. Solving (9) for $y$ with $E(t)=0$ for the chosen parameter values, we get

$$
y_{D H}= \pm x[(x+5.8548)(x+2.6347)(1.6207-x)],
$$

so that the separatrix through the double homoclinic loop passes through $x s_{2}=1.6207$ and $x s_{1}=-2.6347$. There is also a separate branch (not shown) that passes through $x=-5.8548$, whose components tend to $\pm \infty$. Figure 2 shows the potential function $V(x)$, including the two end points $x s_{1}$ and $x s_{2}$ of the separatrix for the double homoclinic loop.

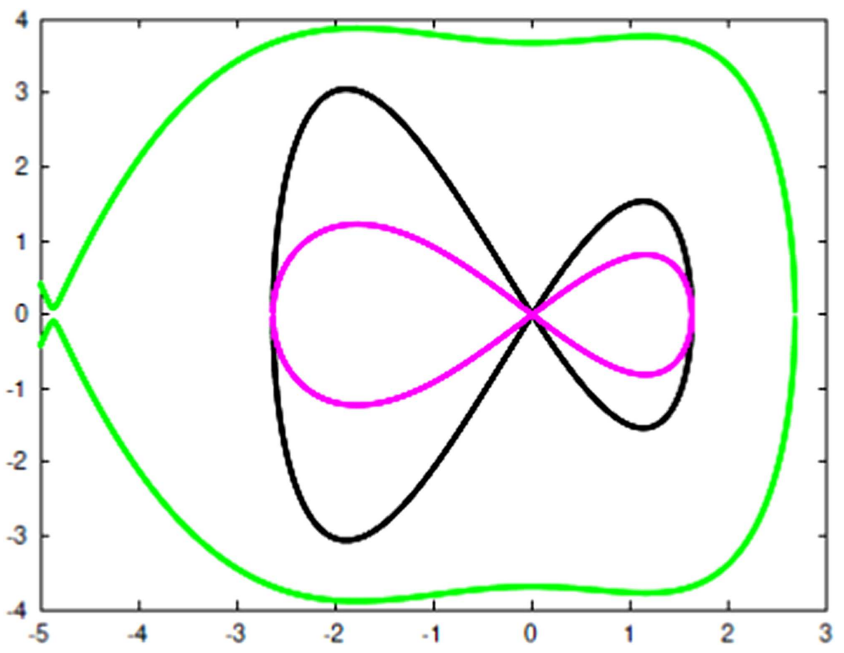

FIG. 2. The double homoclinic loop for the BEH (magenta) passing through the fixed points $(0,0)$ and enclosing the two centers $x_{2}$ and $x_{3}$. There is also a large homoclinic loop (green) passing through $x_{1}$ and enclosing the other fixed points. 
For the large homoclinic loop (green), we got $x=-4.8677$ (twice), $x=2.6711$ (the point at which the large orbit crosses the $y$-axis), and two other complex valued roots.

If we set $\gamma=0$, the model considered by Wang et al., ${ }^{33}$ we no longer get the root $x=-5.8548$, and the result is the larger black double homoclinic loop, shown in Fig. 2. Since $E(t)=0$, Eqs. (10) and (11) give

$$
y=\frac{d x}{d t}= \pm x \sqrt{g(x)}
$$

where

$$
\begin{aligned}
g(x) & =a_{2} x^{2}+a_{1} x+a_{0} \\
& =1-\frac{2 \beta x}{3}-\frac{\delta x^{2}}{2} \\
& =-\left(x-x s_{1}\right)\left(x-x s_{2}\right) .
\end{aligned}
$$

Litak and Borowiec ${ }^{34}$ investigated the case of an asymmetric double homoclinic loop for a potential function of the form Eq. (10) with $\gamma=0$. In Eq. (15), we have taken $x s_{1}$ and $x s_{2}$ to be the end points on the black and magenta separatrices of Fig. 1, given in Eq. (11). Equation (14) uses the expressions for $a_{j}$ from Eq. (10). For (13), we obtain

$$
t=\int \frac{d x}{x \sqrt{g(x)}}=-\frac{1}{\sqrt{a_{0}}} \ln \left|\frac{2 a_{0}+a_{1} x+2 \sqrt{a_{0} g(x)}}{x}\right| .
$$

Provided $a_{0}>0$. Since $a_{0}=0$ [by rearranging Eq. (9)], this is certainly the case here.

We can invert Eq. (17) to find an expression for $x$ and then for $y$ for the double homoclinic loop. Defining $E=\exp t \sqrt{a_{0}}$, taking the exponential of both sides of (17) and rearranging gives, after straightforward algebra, $x=0$ (the saddle point at the origin) and

$$
x_{d h}=\frac{4 a_{0} E}{\left[\left(E-a_{1}\right)^{2}-4 a_{0} a_{2}\right]} .
$$

The derivative $\frac{d x_{d h}}{d t}$ gives the $y$ coordinate for the double homoclinic loop,

$$
y_{d h}=\frac{4 E a_{0}^{3 / 2}\left[a_{1}^{2}-4 a_{0} a_{2}-E^{2}\right]}{\left[\left(E-a_{1}\right)^{2}-4 a_{0} a_{2}\right]} .
$$

\section{A. The unforced BEH system}

For the full BEH with $\gamma$ reinstated, we can find a solution for $x_{d h}$ in terms of Jacobi Elliptic functions of the first and third kinds and involving inverse trigonometric sine functions. We omit their expressions here as not being very instructive. There is, also, no simple equation for the large green homoclinic loop, shown in Fig. 2. Instead, some level sets, corresponding to a potential function $V(x)$ for the full BEH system, are shown in Figs. 3 and 4.

In the absence of external forcing (so that $\Phi(t)=0$ ), the equilibrium states are given by $y=0, z=0$, and $x . s$ a solution to $F_{h}(x)=0$. We, therefore, obtain the trivial equilibrium $x=0$, together with the three nontrivial equilibrium states obtained in the Hamiltonian limit, namely, a saddle point and two centers. For the given set of parameter values, these are the roots of the RHS of

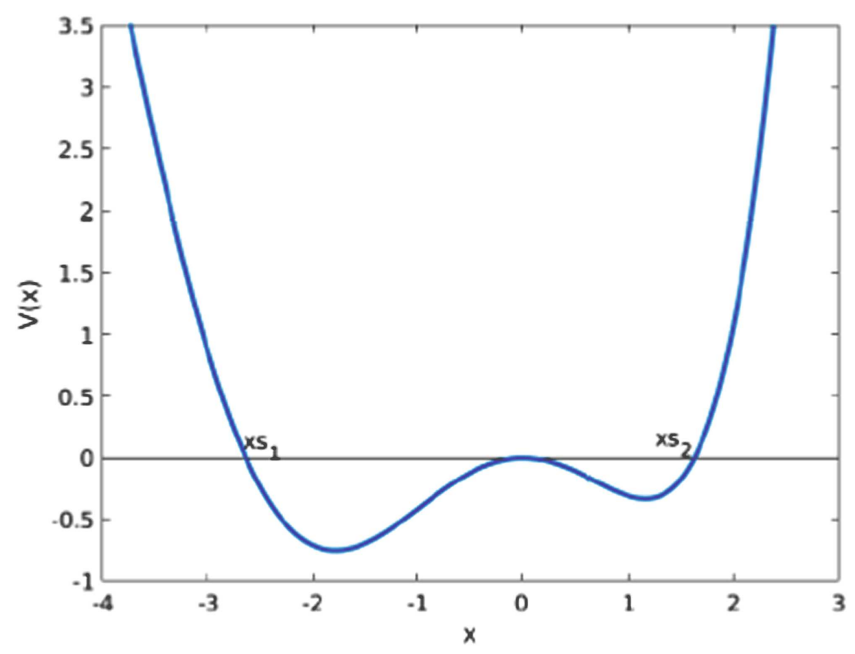

FIG. 3. The potential function $V(x)$ for the BEH with the end points $x s_{1}$ and $x s_{2}$ of the separatrix of the double homoclinic loop.

Eq. (17). The linear stability of the equilibrium state is determined by the eigen spectrum of the characteristic equation,

$$
\lambda^{3}+\Delta_{2} \lambda^{2}+\Delta_{1} \lambda+\Delta_{0}=0,
$$

where

$$
\Delta_{2}=2 \xi+\alpha,
$$

$$
\Delta_{1}=2 \xi \alpha+\kappa-G_{x},
$$$$
\Delta_{0}=-\alpha G_{x},
$$

$$
G_{x}=1-2 \beta x-3 \delta x^{2}-4 \gamma x^{3} \text {. }
$$

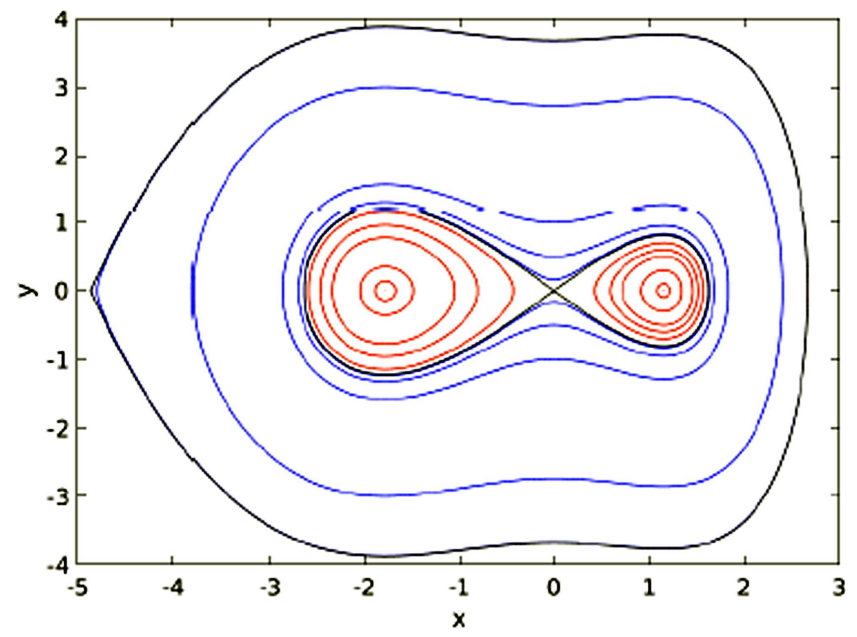

FIG. 4. Some level sets for the potential function $V(x)$ for the BEH. The separatrix for the double homoclinic loop and the large homoclinic loop are shown as black curves. 
TABLE I. Equilibrium points and its stability.

\begin{tabular}{lcc}
\hline \hline Equilibrium points & Eigen values & Stability \\
\hline$E_{0}=[0 ; 0 ; 0]$ & {$[0.9104 ;-1.0962 ;-0.4073]$} & Saddle node \\
$E_{1}=[-4.8675 ; 0 ; 0]$ & {$[2.9155 ;-3.1020 ;-0.4066]$} & Saddle node \\
$E_{2}=[-1.781 ; 0 ; 0]$ & {$[-0.0935 ; \pm 1.2673 i ;-0.4061]$} & Stable focus \\
$E_{3}=[1.1535 ; 0 ; 0]$ & {$[-0.0935 ; \pm 1.4252 i ;-0.4061]$} & Stable focus \\
\hline
\end{tabular}

For the trivial equilibrium state $x=0, G_{x}=\alpha$. This means there are no steady bifurcations from the trivial equilibrium. Moreover, by substituting $\lambda=i \omega$ into Eq. (19), it is straightforward to show that there are also no possible Hopf bifurcations, since this would require $\Delta_{2}+\alpha=0$ : not possible when all parameters are positive. Codimension one steady state bifurcations can occur when $\Delta_{0}=0$ for the nontrivial saddle point at $\gamma=0.139$ (keeping all remaining parameter values at their prescribed values). The condition for a Hopf bifurcation, $\Delta_{0}-\Delta_{1} \Delta_{2}=0$, is not possible since this would require

$$
\omega^{2}=\frac{\alpha G_{x}}{2 \xi+\alpha}=2 \xi \alpha+\kappa-G_{x} .
$$

Numerical integrations show that these two criteria for $\omega^{2}$ cannot be simultaneously satisfied.

Moreover, numerical integrations for the unforced system also show that the only stable states are steady states; we found no evidence of periodic solutions. When the $z$-dependence is present, the saddle point $x_{1}$ remains a saddle, while the two centers $x_{2}$ and $x_{3}$ become stable foci.

For the parameter values $\xi=0.0933 ; \delta=0.5495 ; \alpha=0.4065$; $\beta=0.1 ; \gamma=0.1 ; \kappa=0.00184$, the equilibrium points and corresponding eigen values are calculated and presented in Table I for understanding the stability.

The system shows a chaotic attractor for the following parameter values, $A_{1}=0.5, \omega_{1}=1, A_{2}=1, \omega_{2}=\frac{\sqrt{5}-1}{2}, \xi=0.0933$, $\delta=0.5495, \beta=0.1, \gamma=0.1, \kappa=0.00184, \alpha=0.4065$ for the initial condition $\{1,0,1\}$. The $2 \mathrm{D}$ phase portraits are given in Fig. 5 , where (a) represents the state phase portrait between $\mathrm{Y}$ and Z, (b) represents the state phase portrait between $\mathrm{X}$ vs $\mathrm{Y}$, and (c) represents the state phase portrait between $\mathrm{X}$ vs $\mathrm{Z}$, respectively.

\section{BIFURCATION AND LYAPUNOV SPECTRUM}

We investigated the bifurcation property for two scenarios: case (1) the system under periodic excitation and case (2) the system under quasi-periodic excitation. We used Runge-Kutta numerical method for simulating the results. We considered the parameter values: $A_{1}=0.5, A_{2}=1, \xi=0.0933, \delta=0.5495, \beta=0.1, \gamma=0.1$, $\kappa=0.00184, \alpha=0.4065$, and initial condition $\{1,0,1\}$. For investigating the bistability nature of the system under mentioned excitation conditions, we provided the bifurcation plots using forward continuation (plotted with blue dots) and backward continuation (plotted with red dot), and the corresponding Lyapunov exponent spectrum is calculated using Wolf algorithm ${ }^{35}$ and plotted for a finite time of $20000 \mathrm{~s}$.

\section{Case (1): Under periodic excitation}

First, we considered the system (5) is supplied with periodic excitation $\Phi(t)=F \sin (\omega t)$ and the response of the system is noted and presented as a bifurcation plot. In Fig. 6, we could observe the bistability phenomena which are considered dangerous for mechanical systems. We varied the parameter $\omega$ for the range of 0.9-1.3. For detailed analysis, we provided the bistable regions in Fig. 6(b) and the corresponding Lyapunov exponent spectrum for forward and backward continuation also presented in Figs. 6(c) and 6(d). From the bifurcation plots, we can observe the system holds the multistability property. ${ }^{36-39}$

Case (2): Under quasi-periodic excitation

In this case, we considered the system (5) is supplied with quasi-periodic excitation; real systems that are mostly influenced with different frequencies for simplification purpose only are considered as single frequency, i.e., periodic excitation. In order to analyze the system with quasi-periodic excitation, we considered the parameter values in Eq. (5) to be $A_{1}=0.5, \omega_{1}=1, \omega_{2}=\frac{\sqrt{5}-1}{2}$, and $A_{2}=1$. The influence of excitation frequency plays a vital role in behavioral analysis. We derived the bifurcation plots for frequency

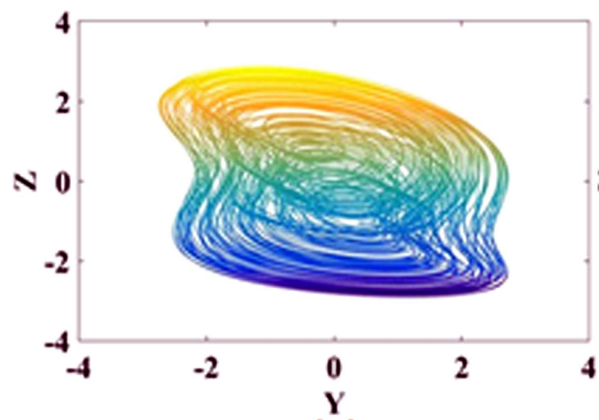

(a)

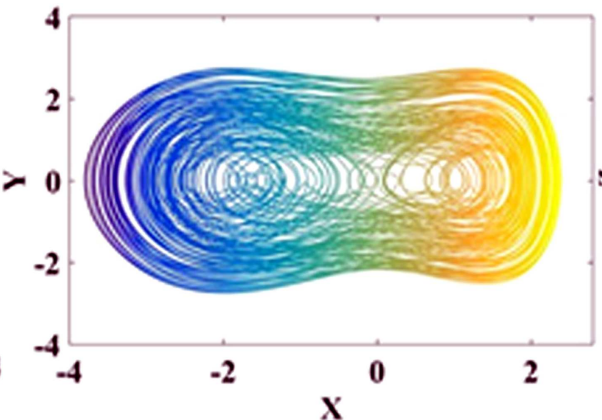

(b)

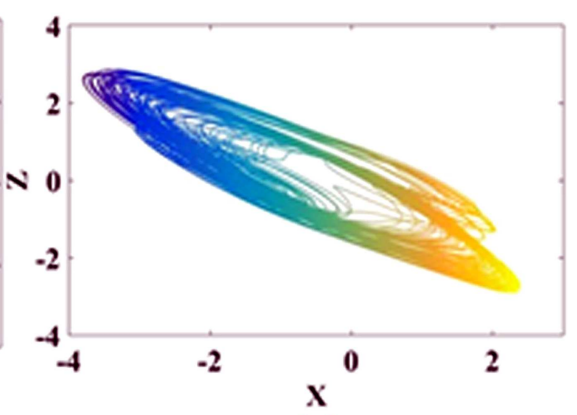

(c)

FIG. 5. 2D phase portrait of system (6) for the parameter values $A_{1}=0.5, \omega_{1}=1, A_{2}=1, \omega_{2}=\frac{\sqrt{5}-1}{2}, \xi=0.0933, \delta=0.5495, \beta=0.1, \gamma=0.1, \kappa=0.00184$, $\alpha=0.4065$ and initial condition $\{1,0,1\}$ (a) $Y$ vs Z, (b) X vs $Y$, and (c) $X$ vs Z. 

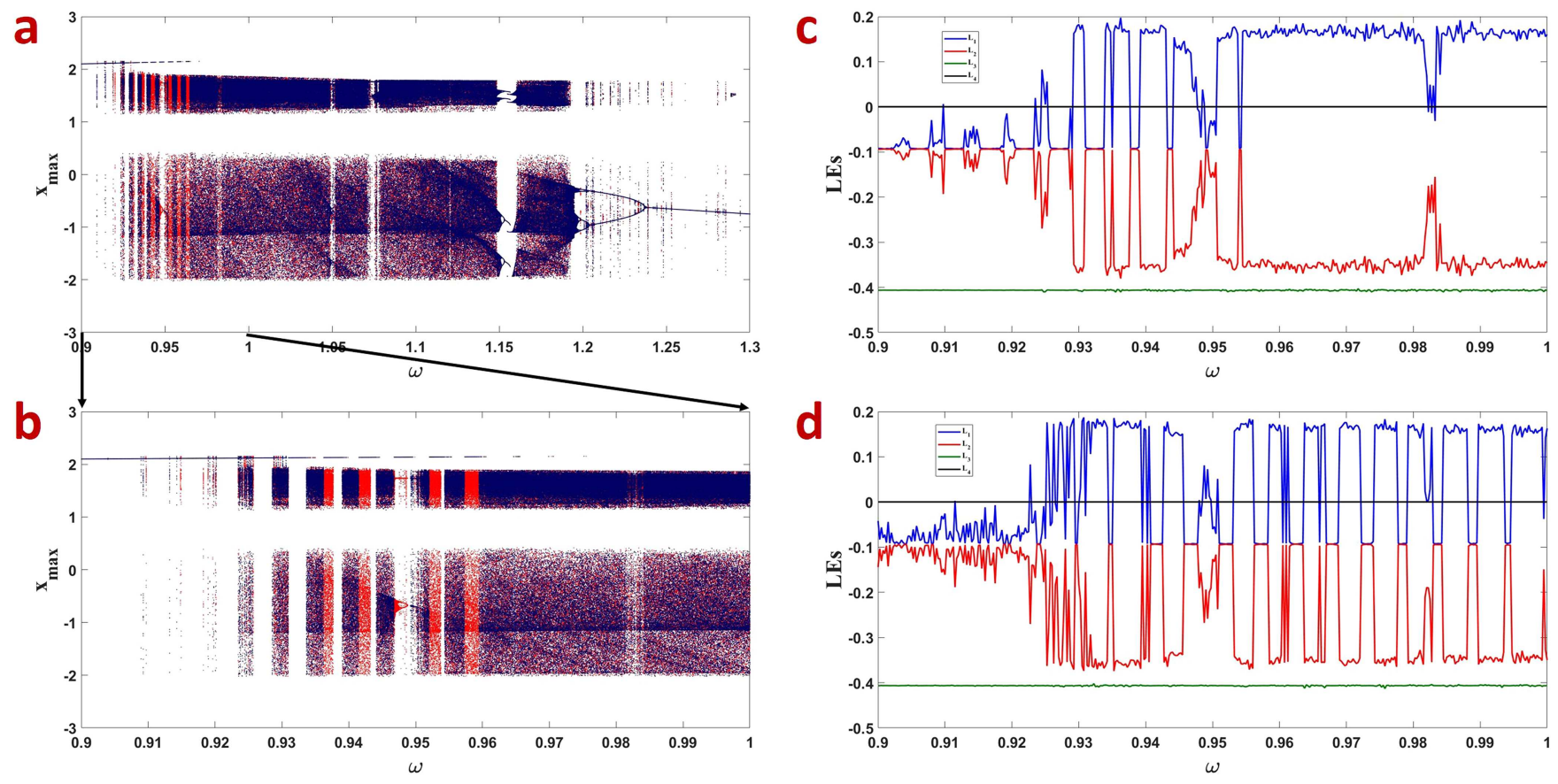

FIG. 6. Bifurcation plot and the corresponding Lyapunov exponents of parameter $\omega_{1}$ variation. (Periodic excitation) (a) Represents the bifurcation plot of $0.9 \leq \omega_{1} \leq 1.3$, (b) represents the bifurcation plot of $0.9 \leq \omega_{1} \leq 1$, (c) represents Lyapunov exponents spectrum for (a), and (d) represents Lyapunov exponents spectrum for (b), respectively.

range $0 \leq \omega_{1} \leq 3$. The corresponding Lyapunov exponent spectrum also presented. Comparing with periodic excitation (Fig. 6), we could observe that there is no limit cycle oscillation as in Fig. 7. Chaotic attractor and torus were identified, and the corresponding Lyapunov exponent spectrum confirms it. We could observe that the multistability property is vanished during quasi-periodic excitation.

\section{NETWORK DYNAMICS OF BEH}

The local behavior of the BEH with periodic and quasi-periodic excitations shows some interesting dynamical behaviors but energy harvesters will normally be applied in large networks. Hence, we consider $\mathrm{N}$ coupled $\mathrm{BEH}$ whose mathematical model is shown in Eq. (25)

$$
\begin{gathered}
\dot{x}_{i}=y_{i}+\sigma \sum_{j=1}^{N} C_{i j} x_{j}, \\
\dot{y}_{i}=\Phi(t)-2 \xi y_{i}+x_{i}-\delta x_{i}^{3}-\beta x_{i}^{2}-\gamma x_{i}^{4}+\kappa z_{i}, \\
\dot{z}_{i}=-y_{i}-\alpha z_{i} .
\end{gathered}
$$

The term $\sigma$ defines the coupling constant and the connection between the nearby nodes is defined by the connection matrix $C_{i j}$. The external excitation is defined by $\Phi(t)=A_{1}\left[\sin \left(\omega_{1} t\right)\right.$ $\left.+A_{2} \sin \left(\omega_{2} t\right)\right]$, where $\omega_{1}=1$ is the frequency of the periodic term while $\omega_{2}=\frac{\sqrt{5}-1}{2}$ is the golden mean contributing to the quasiperiodic excitation. We have considered two different cases for discussion depending on the type of excitation applied to the nodes in the network.

\section{A. Network behavior with periodic excitation}

In this case, we consider $A_{2}=0$, we now apply a periodic excitation to the nodes in the network and for simulation we consider the parameters as $A_{1}=0.5, \omega_{1}=1, \xi=0.0933, \delta=0.5495$, $\beta=0.1, \gamma=0.1, \kappa=0.00184, \alpha=0.4065$ and random initial conditions are chosen. We have used the RK4 method to solve the system (25) with the step size of 0.01 and a total simulation time of $3000 \mathrm{~s}$. In Fig. 8, we have shown the spatiotemporal behavior of the network for coupling strengths $\sigma \leq 0.015$ for which we could see that the nodes are in complete incoherency. We have also plotted the instantaneous state variable value of $x$ measured at the end of simulation. For $\sigma=0.015$, the nodes try to achieve synchronization and lead us to a clue to check for chimeras by increasing coupling.

Now, we increased the coupling to $\sigma=0.018$ to identify the existence of coherent and incoherent nodes in the network. In Fig. 9, we could see that most of the nodes try to achieve coherency and some nodes are still in the complete incoherent state. This confirms the existence of chimeras in the network. It should be noted that only if all the $\mathrm{BEH}$ nodes are in the coherent state, the energy efficiency will be more, and such chimeras will result in residue current in the BEH nodes which could damage the node permanently. Such chimeras are seen in the network for $\sigma<0.03$ but we have only shown spatiotemporal behavior of selected values of $\sigma$. 

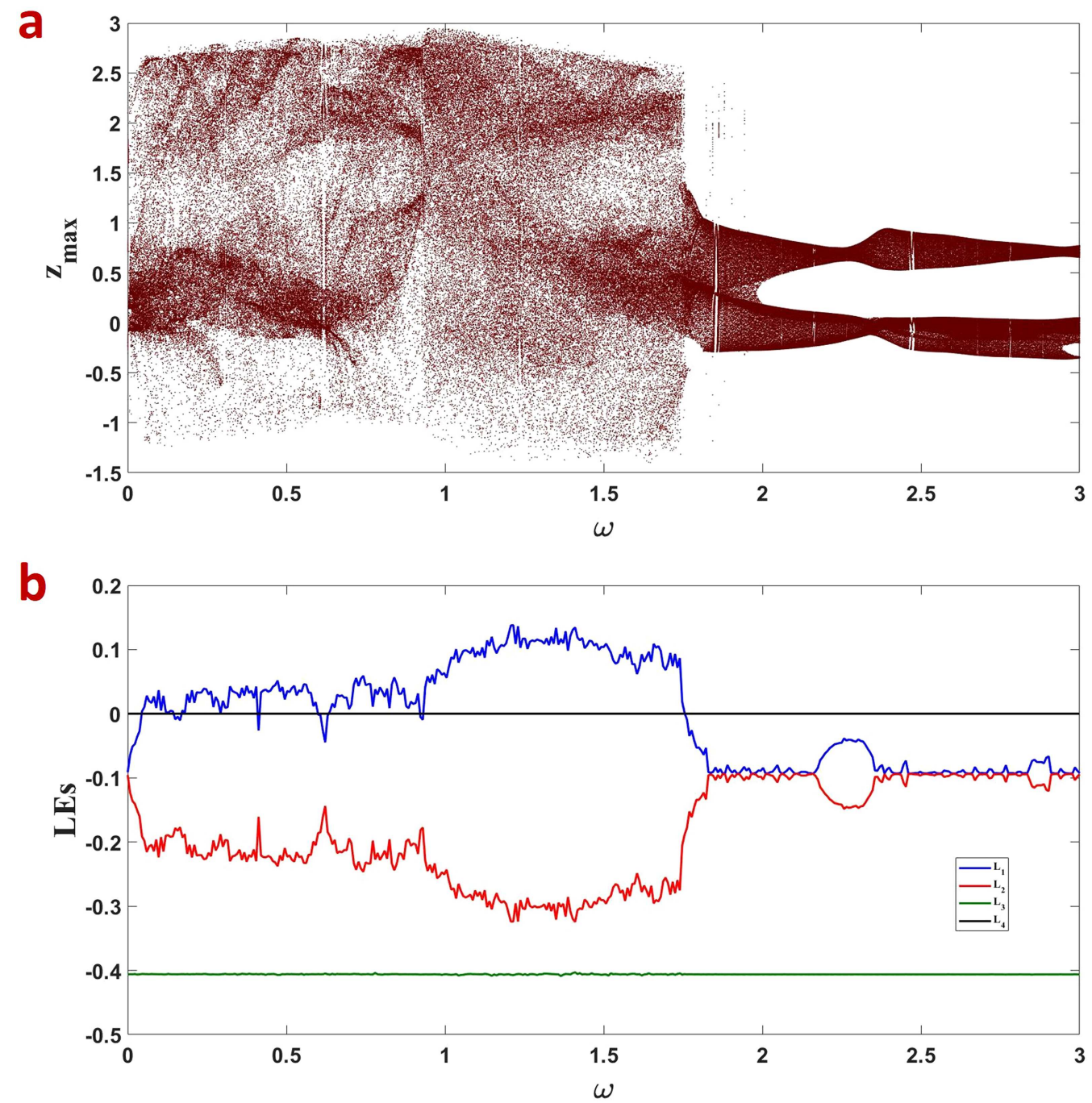

FIG. 7. Bifurcation analysis of system (5) under quasi-periodic excitation, (a) represents the bifurcation plot for $0 \leq \omega_{1} \leq 3$ and (b) represents the Lyapunov exponent spectrum for $0 \leq \omega_{1} \leq 3$.

Further increasing the coupling strength to $\sigma=0.03$, the nodes try to achieve synchronization in small clusters. Though such phenomenon of cluster synchronization is not uncommon in spiking networks, its undesirable in such networks where complete synchronization is mandatory. In other words, we need all the BEH nodes in the network to operate in a single frequency or at least in coherency to maximize the energy efficiency of the harvesters. In Fig. 10, we have shown several cluster synchronization conditions for various values of $\sigma$.

We have used re-occurrence plots to identify different regimes of synchronization in the network. The re-occurrence plots are calculated by finding the Euclidean distance between $x_{i}$ and $x_{j}$ where $i . j \in[1, N]$. In Fig. 11, we have shown the re-occurrence plots and the absence of structures in the plot for $\sigma=0.001$ shows that there are no coherent nodes in the network. For $\sigma=0.01$, we could see small structures formed in the network confirming the emergence of chimeras. For the remaining values of $\sigma<0.03$ shown in the plots, we could see majority of blue and red regions. The blue region shows coherent oscillators and the red shows the incoherent oscillators. The presence of other colors in these plots confirms the existence of multiple intermediate nodes which neither belong to the red and nor to the blue regions confirming different incoherent frequencies in the network. But when $\sigma>0.03$ we could note only blue and red dominant confirming different clusters of synchronizations.

Though we could achieve cluster synchronization in the network as shown in Fig. 11, we could not reach complete synchronization. For values of coupling $\sigma>0.1$, the network goes in to unbounded states and thus we could not use the coupling 

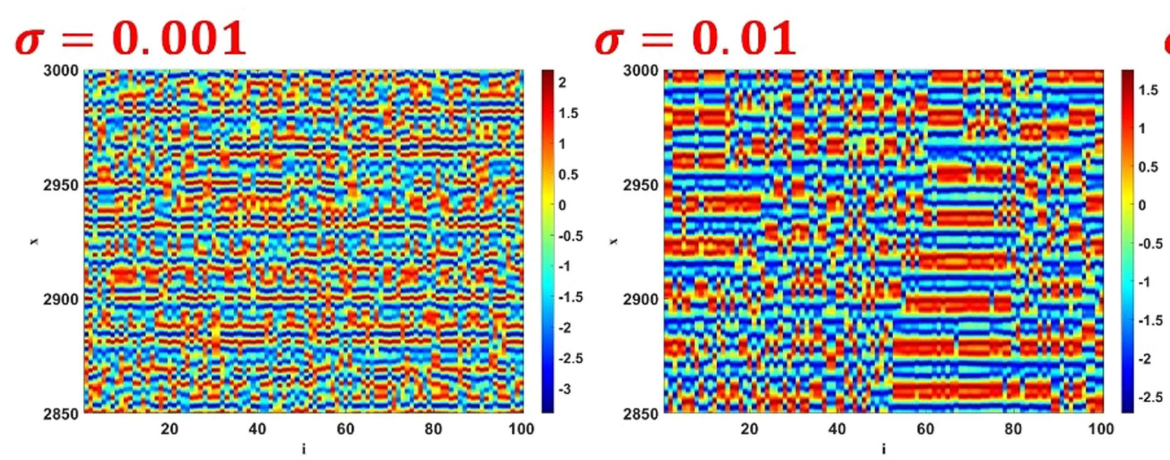

$\sigma=0.015$
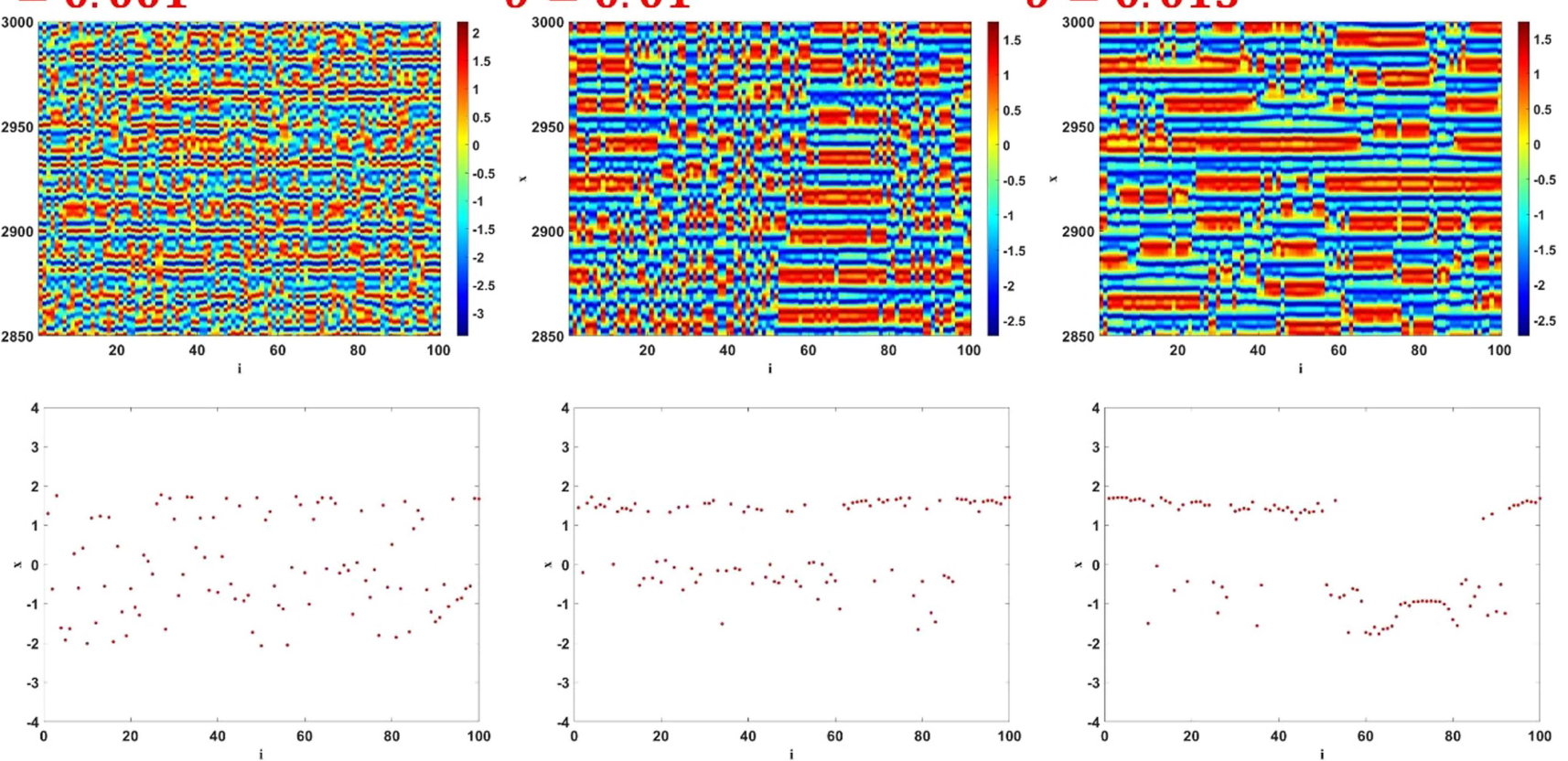

FIG. 8. The collective behavior of the network (1) for different values of the coupling coefficient $(\sigma)$ considering a periodic excitation. The plots confirm that the nodes are asynchronous.

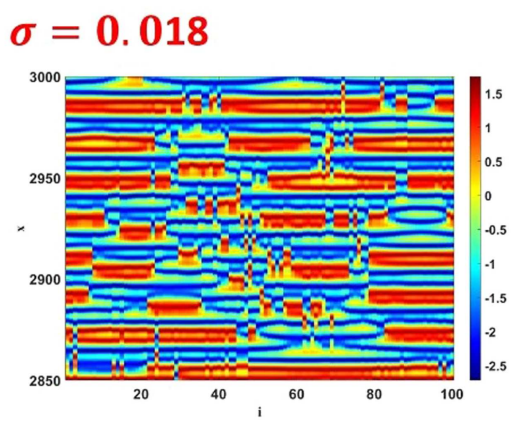

$$
\sigma=0.02
$$
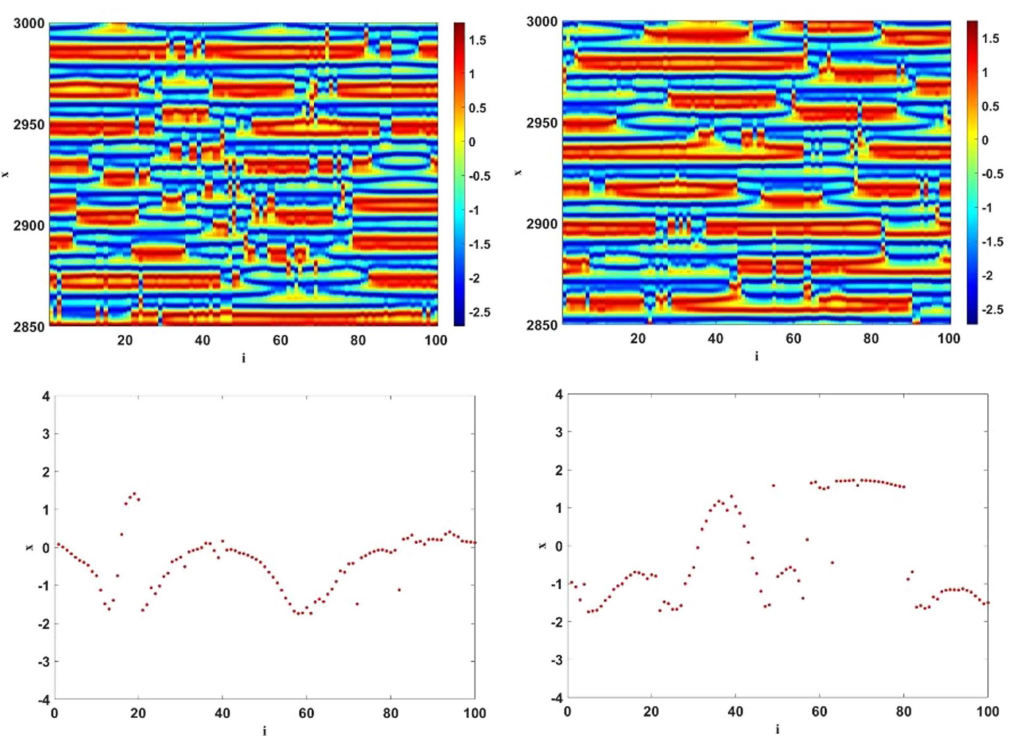

$$
\sigma=0.022
$$
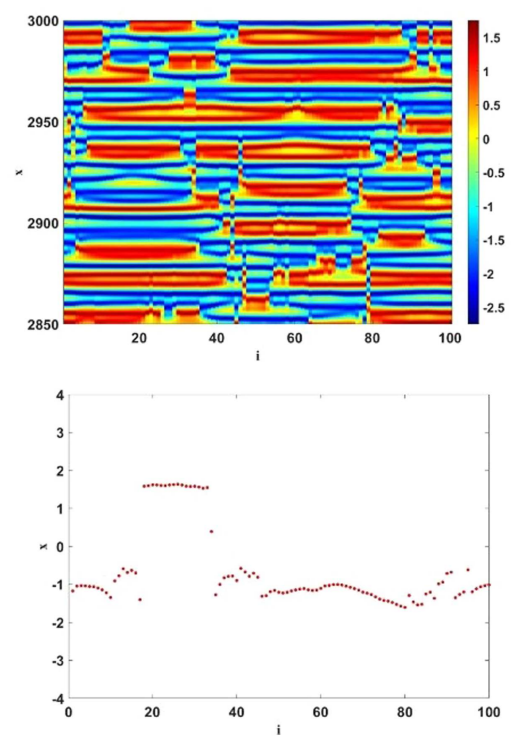

FIG. 9. The collective behavior of the network (25) for different values of the coupling coefficient $(\sigma)$ considering a periodic excitation. The plots confirm that the nodes are showing both synchronous and asynchronous nodes confirming the existence of chimeras. 

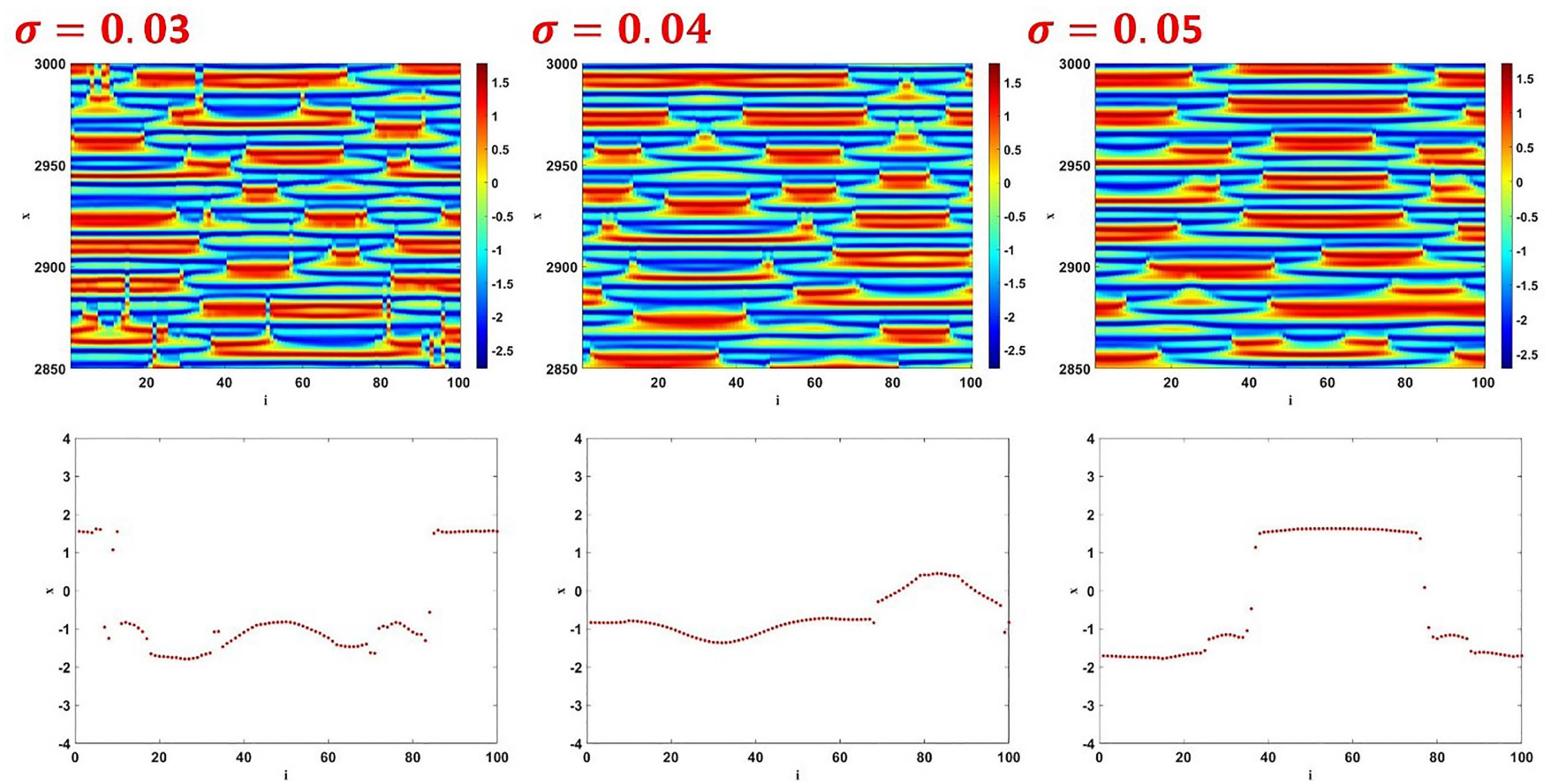

FIG. 10. The collective behavior of the network (25) for different values of the coupling coefficient $(\sigma)$ considering a periodic excitation. The plots confirm several clusters of synchronized BEH nodes in the network.

strength to achieve synchronization. As the $\mathrm{BEH}$ is externally excited by the periodic force, our interest now is on the amplitude of the periodic force which can be properly tuned to achieve complete synchronization. In Fig. 12, we have shown the spatiotemporal plots for the amplitude values $A_{1}=[0.4,0.45,0.5]$ and the plots confirm the emergence of chimeras in the network. Though for $A_{1}=0.5$, the incoherent nodes are very less confirming that the nodes are moving to complete synchronization.

In Fig. 13, we have shown the complete synchronization of the nodes achieved through the tuning of the amplitude of the external excitation. We could note that for $A_{1}<0.4$ and $A_{1}>0.6$ the nodes attain complete synchronization and achieve the same frequency of operation. Thus, we could achieve the maximum efficiency from the energy harvesters when connected to a network. But it has been shown in the literature that non-chaotic $\mathrm{BEH}^{34}$ can be productive in energy harvesting but as seen from the re-occurrence plot (Fig. 12 right most) for $A_{1}=0.3$, the nodes are no more chaotic and show periodic behaviors and for $A_{1}=0.7$ the nodes are chaotic and are completely synchronized as there Euclidean distance is very low in $10^{-13}$. Thus, we recommend an amplitude value of $A_{1}=0.3$ to achieve complete synchronization in the periodically excited $\mathrm{BEH}$ network.

\section{B. Network behavior with quasi-periodic excitation}

In this case, we consider $A_{1}=0.5$, we now apply a quasiperiodic excitation to the nodes in the network by considering
$A_{2} \neq 0, \omega_{2}=\frac{\sqrt{5}-1}{2}$ with the other parameters as $\omega_{1}=1, A_{2}=1$, $\xi=0.0933, \delta=0.5495, \beta=0.1, \gamma=0.1, \kappa=0.00184, \alpha=0.4065$. The other setting for simulations is similar to Sec. $V$ A. As we have earlier shown that the coupling coefficient could not be tuned to achieve complete synchronization (Sec. V A), we verified the same for quasi and could again confirm that $\sigma$ cannot be tuned for synchronization in the quasi-periodic case. Hence, we have not provided the discussion on $\sigma$ as it will be redundant. We focus our discussion on the amplitude of the quasi-periodic term $\left(A_{2}\right)$ and have kept $\sigma=0.025$. In Fig. 14, we have shown the spatiotemporal plots for different values of $A_{2}$ and unlike Fig. 13, we could not find periodic regimes for $A_{2}<1$ and the network shows both coherent and incoherent nodes confirming the existence of chimeras.

To find the amplitude values that could help us achieve complete synchronization with nodes are not in their chaotic regime, we further increase the value of $A_{2}=1.5$ and could observe complete synchronization and the same is the case for $A_{2}=1.8$, presented in Fig. 15. But our interest is to check whether the nodes are not in their chaotic regime while achieving complete synchronization.

As discussed, we must verify which of the amplitude $\left(A_{2}\right)$ can achieve complete synchronization while the $\mathrm{BEH}$ nodes are not chaotic. Hence, we use the re-occurrence plots as shown in Fig. 16. While checking the re-occurrence plots for $A_{2}=1.5$ we could see that the nodes are in synchronization with period- 4 oscillations and for $A_{2}=1.8$, the nodes whose different colors confirming they are chaotic but their Euclidian distance in the range of $10^{-13}$ showing 

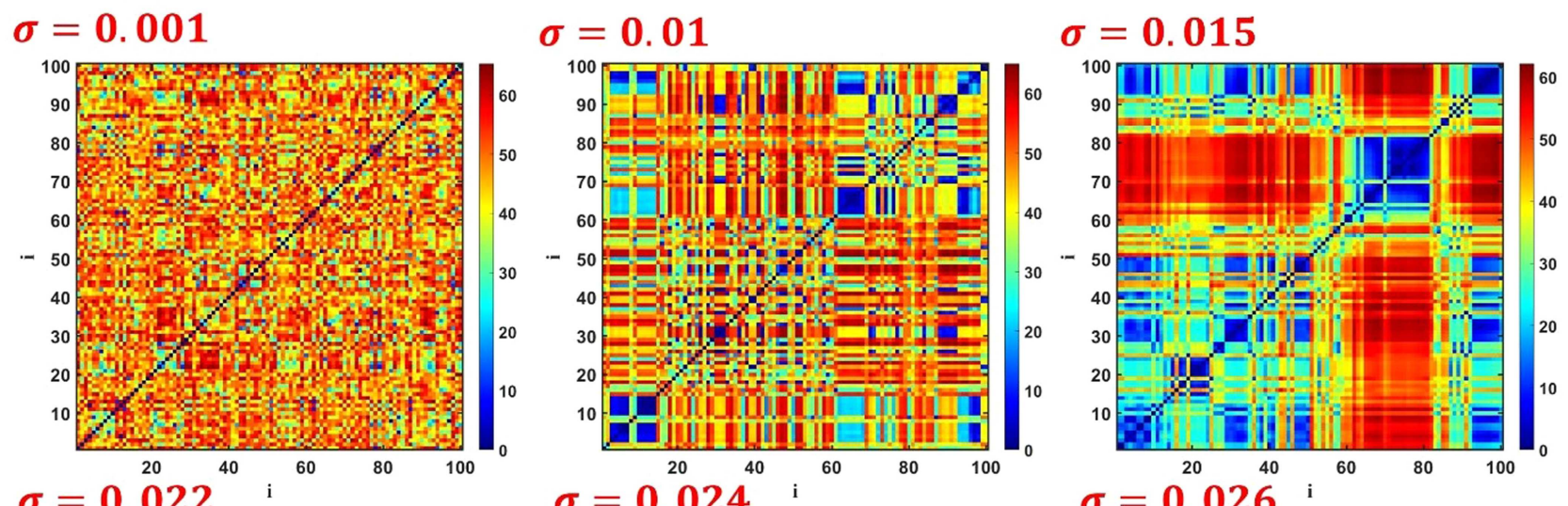

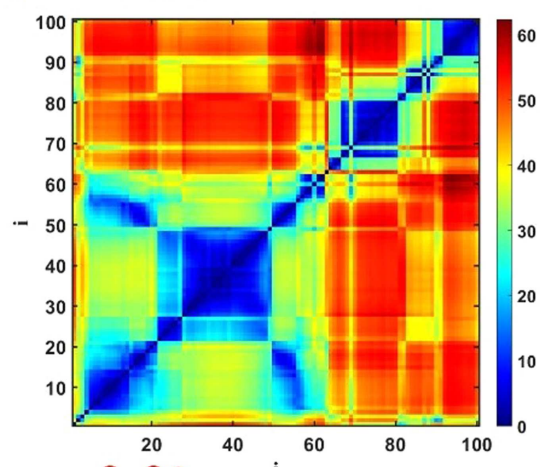

$$
\sigma=0.024
$$

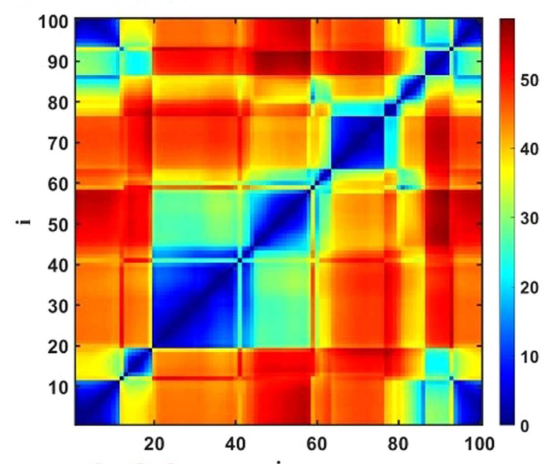

\section{$\sigma=0.04$}
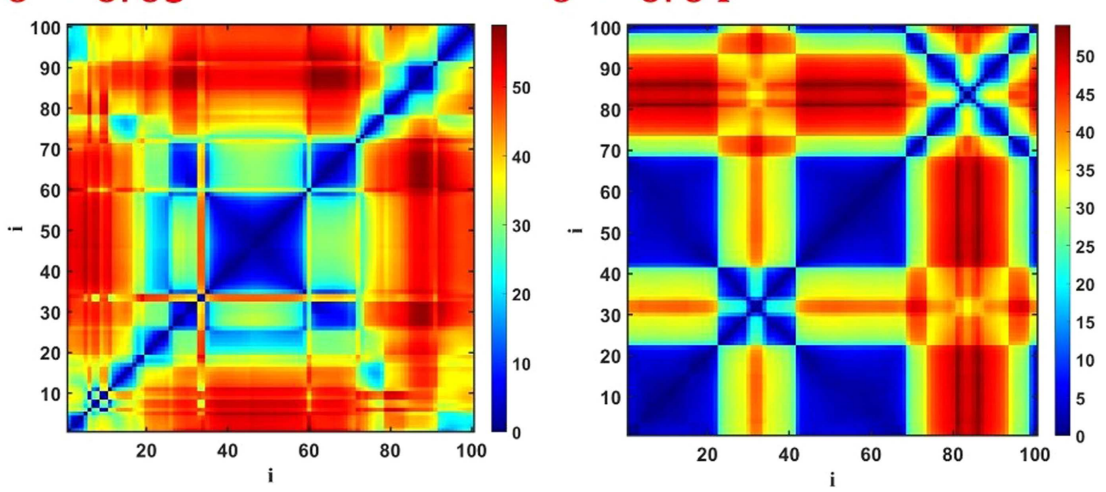

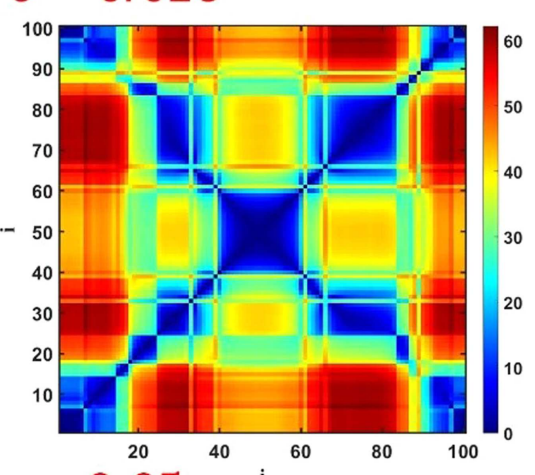

$\sigma=0.05$

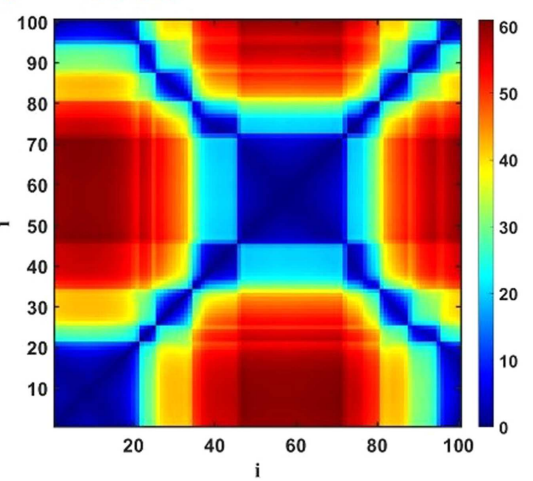

FIG. 11. Re-occurrence plots of the BEH network for different values of the coupling coefficient. The blue regions show the coherent oscillators and the red show the incoherent oscillators.

complete synchronization. Hence, when exposed to quasi-periodic excitation, we could recommend the amplitude combination as $A_{1}=0.5$ and $A_{2}=1.5$ for complete synchronization as for these two amplitude values the nodes are periodic and chaotic oscillations are suppressed.

\section{CONCLUSION}

An energy harvester model with two stable equilibrium points is analyzed considering periodic and quasiperiodic external excitations. The dynamical properties of the model are analyzed and we could show coexisting hidden attractors in the system for periodic excitation. Though local behavior can help us understand the complex oscillations and bifurcation patterns of the bistable energy harvesters, our focus is to investigate its collective dynamics. A mathematical model of a 2D lattice network is constructed whose local dynamics is governed by the bistable energy harvesters. First, a periodic excitation is applied to the nodes and the spatiotemporal behavior is captured. We could observe regions of asynchronous nodes for very low coupling values, while increasing the coupling 
$A_{1}=0.4$
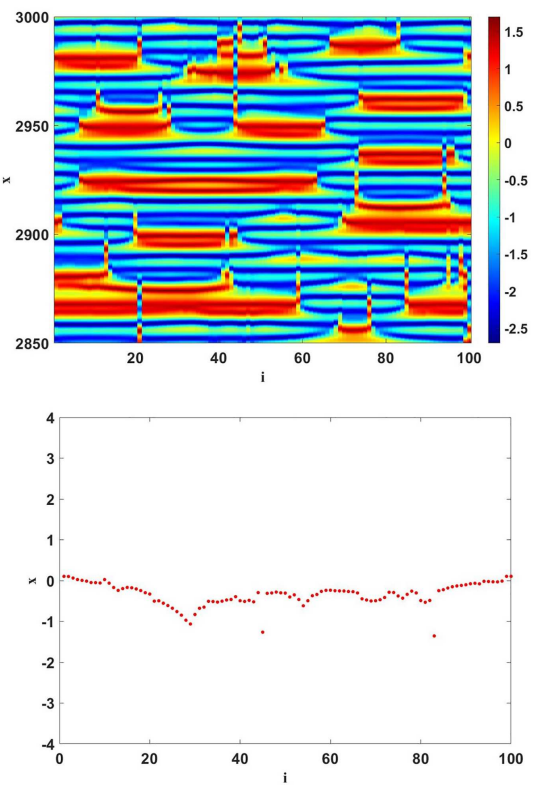

$A_{1}=0.45$
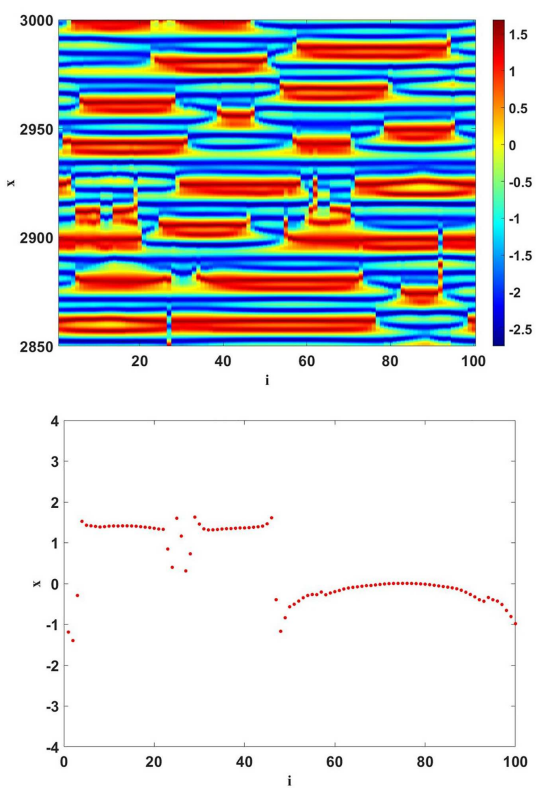

$A_{1}=0.5$
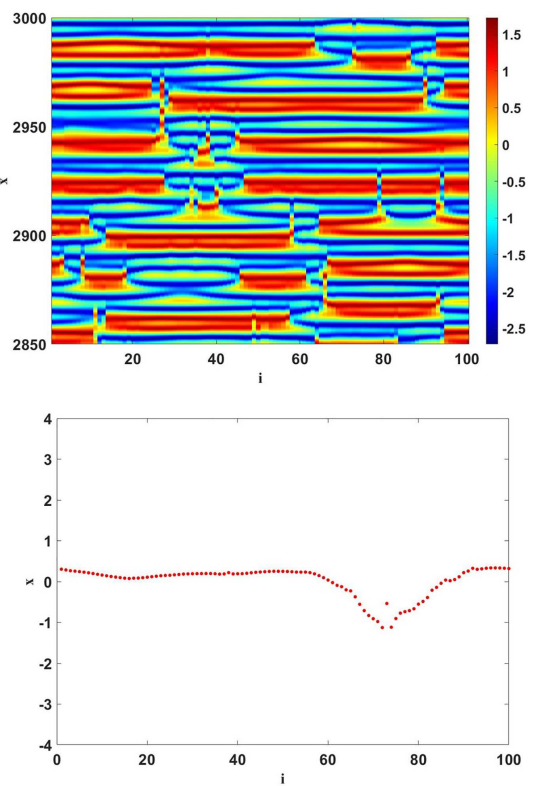

FIG. 12. The collective behavior of the network (1) for different values of the amplitude of the periodic excitation considering $\sigma=0.024$. The plots emergence of chimera states with synchronous and asynchronous nodes.
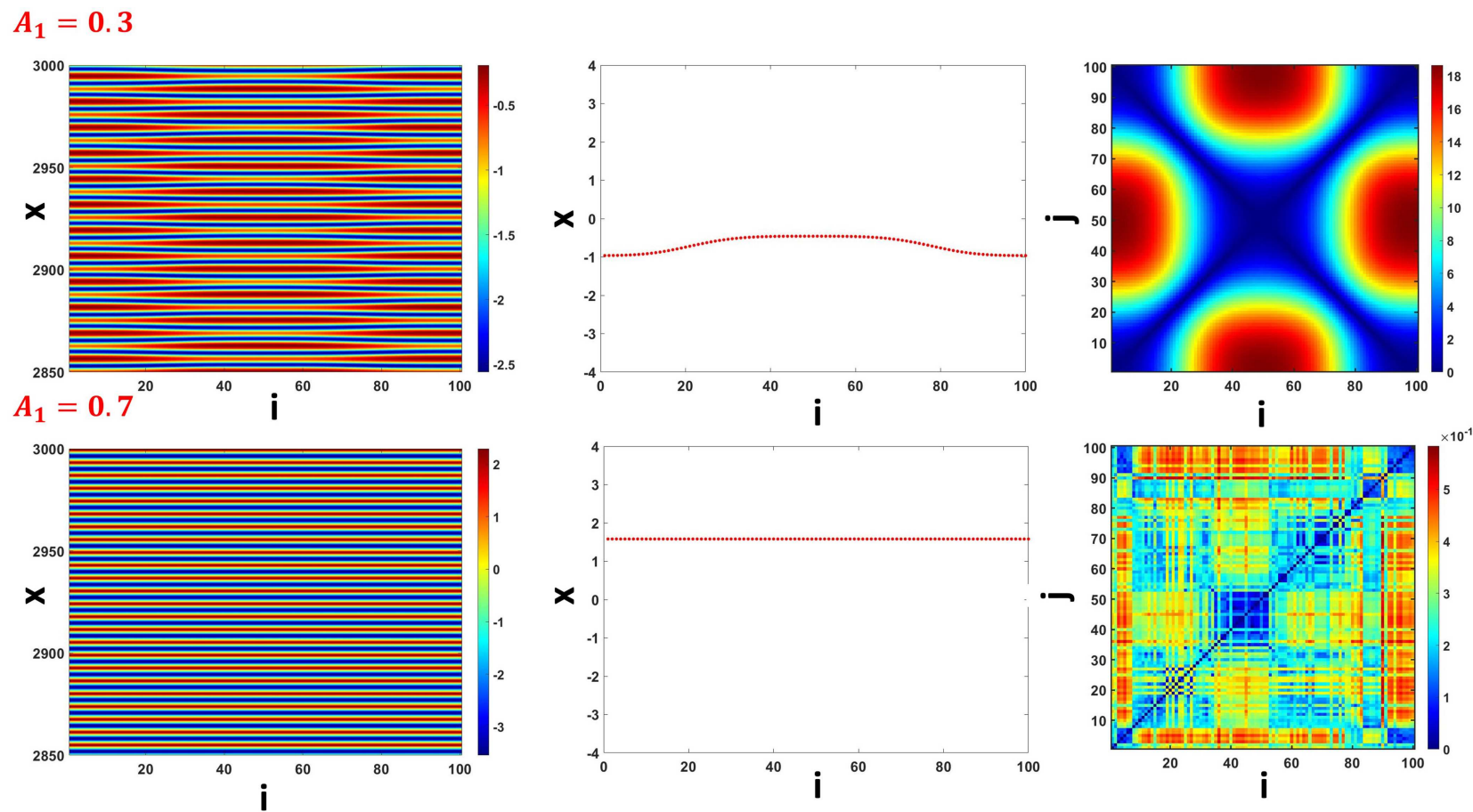

FIG. 13. The collective behavior of the network (25) for different values of the amplitude of the periodic excitation considering $\sigma=0.024$. The plots confirm the nodes achieving complete synchronization. 


$$
A_{2}=0.1
$$
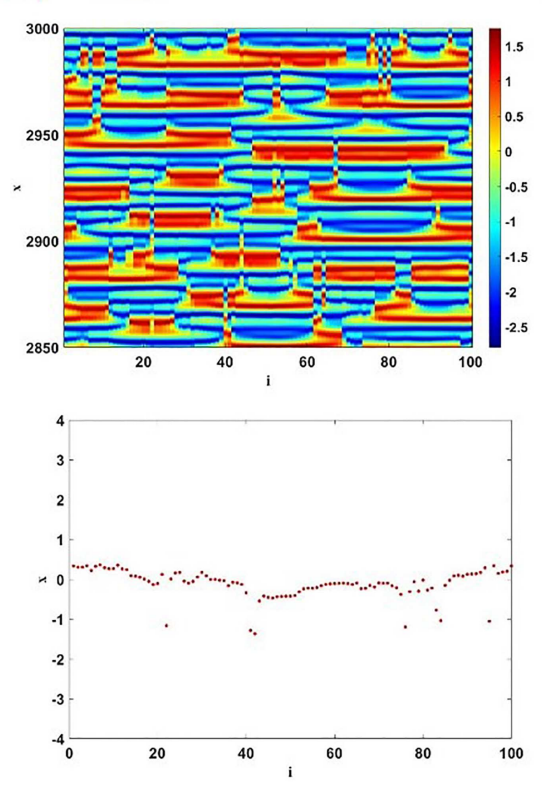

$$
A_{2}=0.5
$$
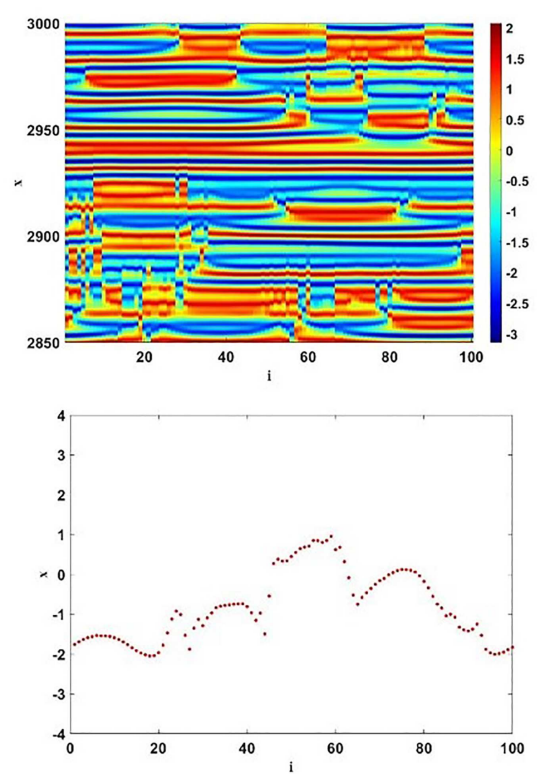

$$
A_{2}=0.8
$$
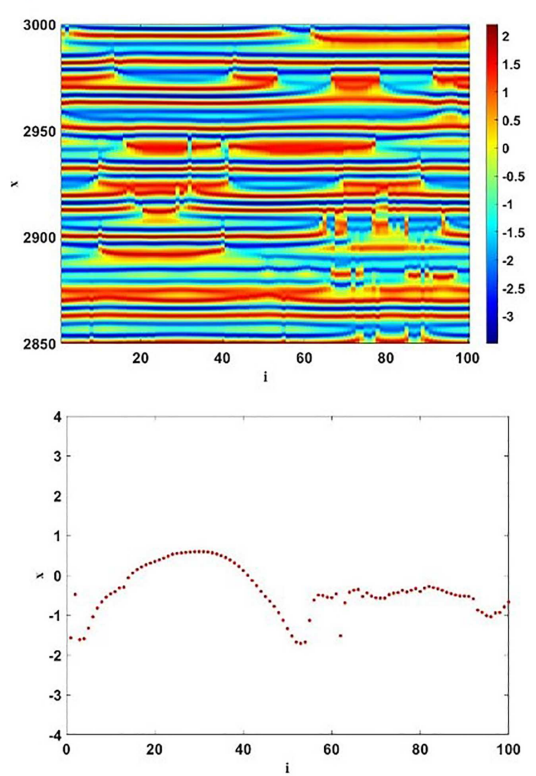

FIG. 14. The collective behavior of the network (25) for different values of the amplitude of the quasi-periodic excitation considering $\sigma=0.025$. The plots confirm the emergence of chimeras.

$$
A_{2}=1
$$
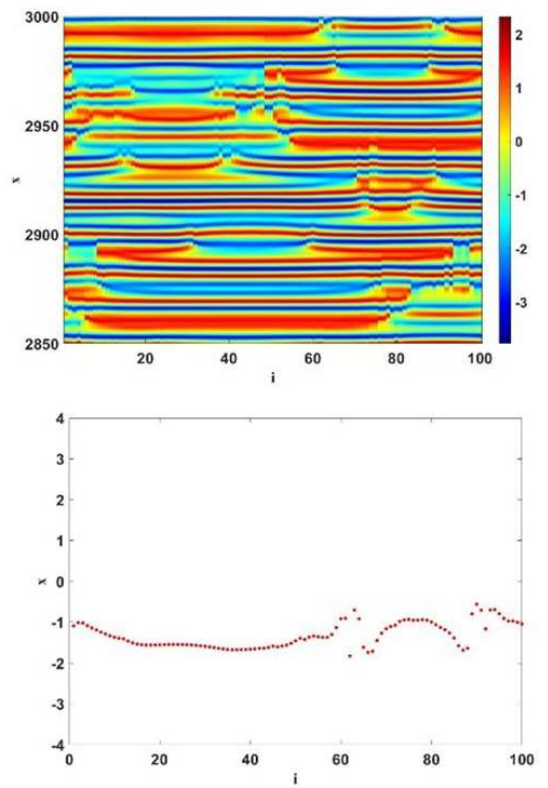

$A_{2}=1.5$
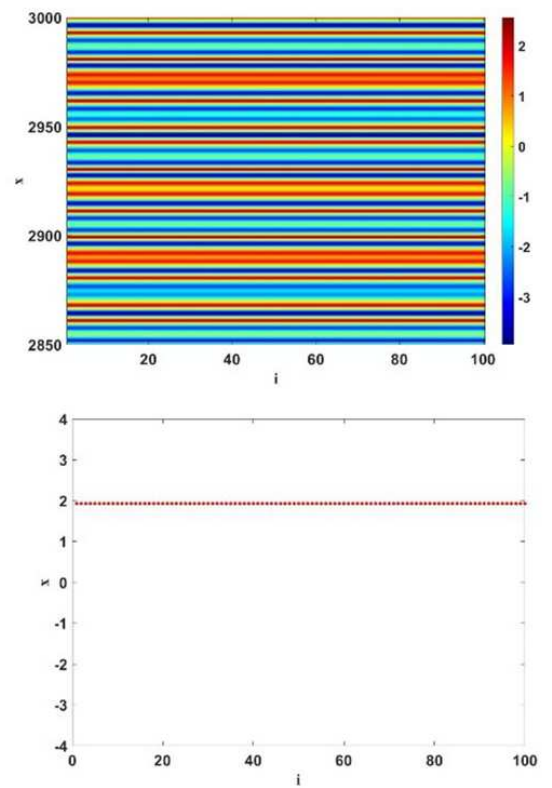

$$
A_{2}=1.8
$$
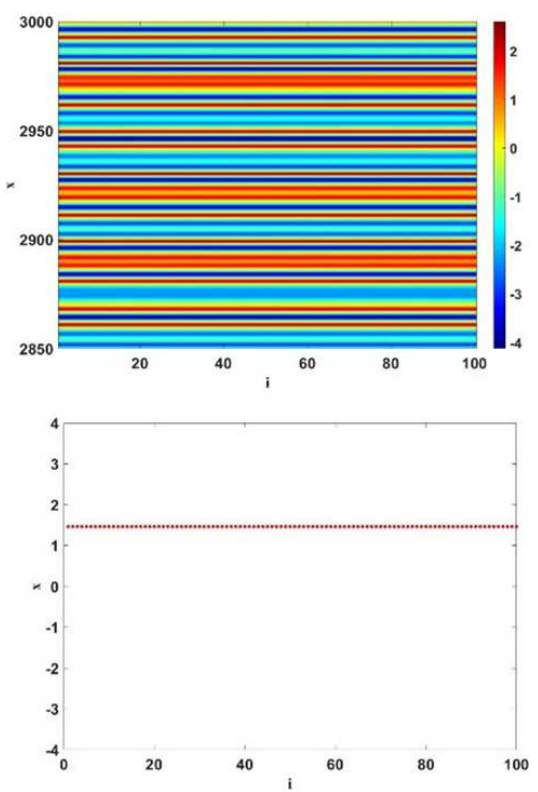

FIG. 15. The collective behavior of the network (25) for different values of the amplitude of the quasi-periodic excitation considering $\sigma=0.025$. The plots confirm nodes achieving complete synchronization for $A_{2}=1.5$ and $A_{2}=1.8$. 
$A_{2}=0.1$
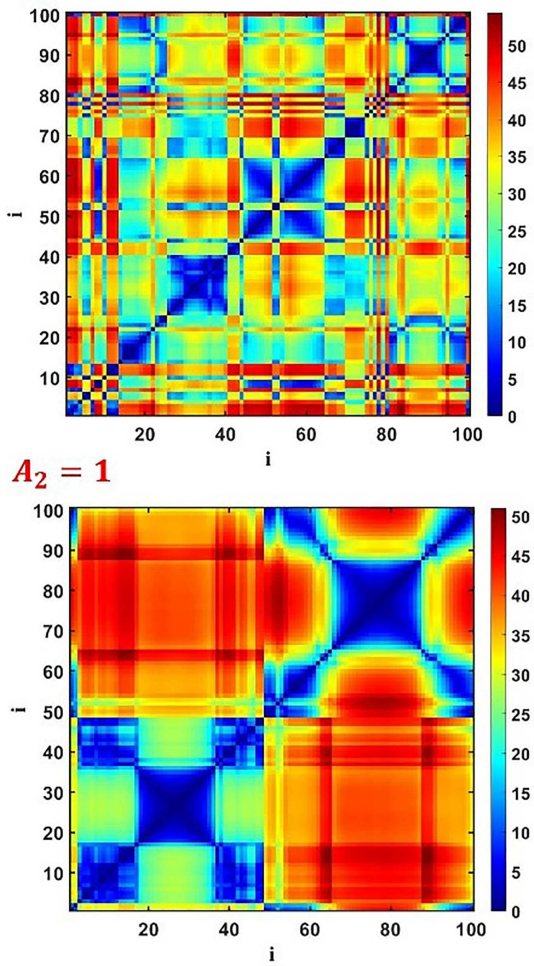

$A_{2}=0.5$

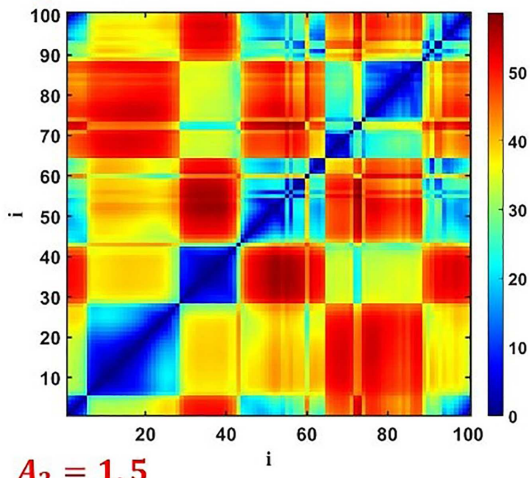

$A_{2}=1.5$

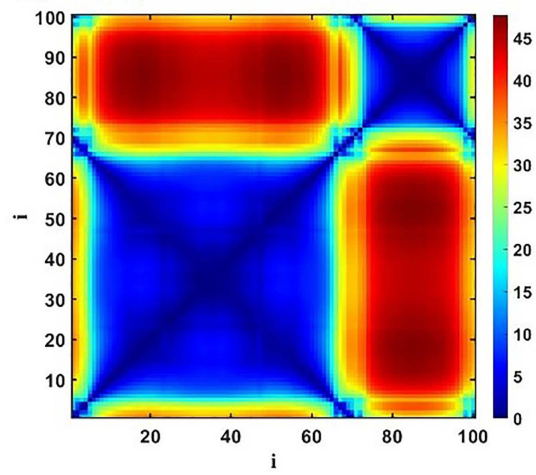

$A_{2}=0.8$
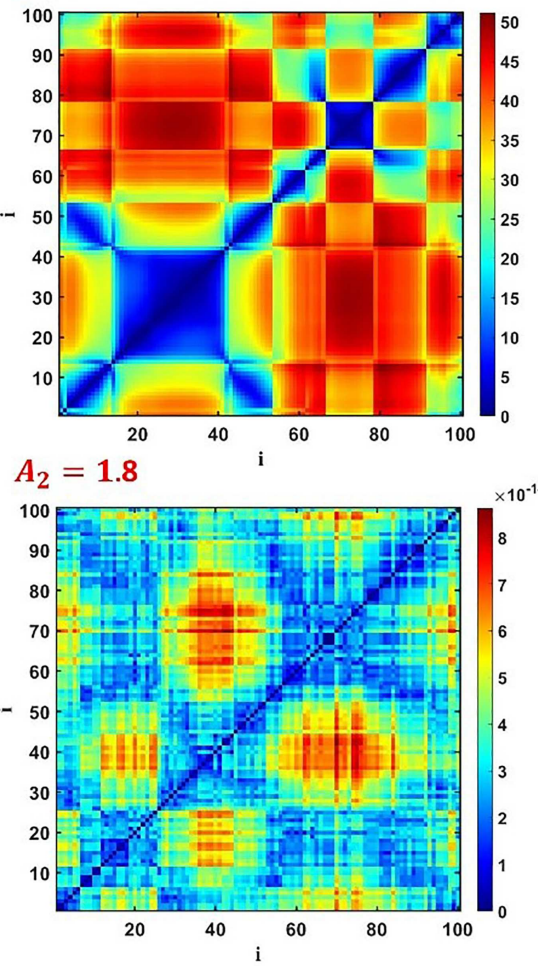

FIG. 16. Re-occurrence plots for different values of $A_{2}$ with the blue regions showing coherent nodes while the other colors showing incoherent nodes.

some nodes are synchronized while some remain incoherent. This confirms the emergence of chimeras. We tried different values of the coupling to observe complete synchronization, but we could observe only cluster synchronization. Hence, we shifted our investigation to the excitation amplitude, and we could observe two ranges of amplitude which could achieve complete synchronization. But our interest is on the amplitude for which the nodes are not in chaos, but the network achieved synchronization. To calculate this, we introduced the re-occurrence plots which is useful in understanding different regimes in a network. From the re-occurrence plots, we could show that for a certain amplitude the nodes are not in chaos, but the network achieved synchronization. Similar studies are conducted for quasiperiodically excited energy harvesters and again we could show some values of the amplitude that can achieve synchronization without disturbing the local complex behavior of the nodes.

\section{ACKNOWLEDGMENTS}

Arthanari Ramesh, Karthikeyan Rajagopal, and Prakash Duraisamy have been partially funded by the Research Grant of the Center for Nonlinear Systems, Chennai Institute of Technology with Reference No. CIT/CNS/2021/RP-017.

The authors declare that they have no conflict of interest.

\section{DATA AVAILABILITY}

The data that support the findings of this study are available from the corresponding author upon reasonable request.

\section{REFERENCES}

${ }^{1}$ A. Erturk, J. M. Renno, and D. J. Inman, "Modeling of piezoelectric energy harvesting from an L-shaped beam-mass structure with an application to UAVs," $J$. Intell. Mater. Syst. Struct. 20, 529-544 (2009).

${ }^{2}$ A. Raghavan and C. E. S. Cesnik, "The shock and vibration digest review of guided-wave structural health monitoring," Shock Vib. Dig. 39, 91-114 (2007).

${ }^{3}$ J. M. Gilbert and F. Balouchi, "Comparison of energy harvesting systems for wireless sensor networks,” Int. J. Autom. Comput. 5, 334-347 (2008).

${ }^{4}$ M. F. Daqaq, R. Masana, A. Erturk, and D. Dane Quinn, "On the role of nonlinearities in vibratory energy harvestingâẮr: A critical review and discussion," Appl. Mech. Rev. 66, 040801 (2014).

${ }^{5}$ S. P. Beeby, M. J. Tudor, and N. M. White, "Energy harvesting vibration sources for microsystems applications,” Meas. Sci. Technol. 17, 175 (2006).

${ }^{6}$ M. Rezaei, S. E. Khadem, and P. Firoozy, "Broadband and tunable PZT energy harvesting utilizing local nonlinearity and tip mass effects," Int. J. Eng. Sci. 118, 1-15 (2017).

${ }^{7}$ S. Zhou, J. Cao, and J. Lin, “Theoretical analysis and experimental verification for improving energy harvesting performance of nonlinear monostable energy harvesters," Nonlinear Dyn. 86, 1599-1611 (2016).

${ }^{8}$ K. Fan, Q. Tan, Y. Zhang, S. Liu, M. Cai, and Y. Zhu, "A monostable piezoelectric energy harvester for broadband low-level excitations," Appl. Phys. Lett. 112(12), 12390 (2018). 
${ }^{9}$ V. R. Challa, M. G. Prasad, Y. Shi, and F. T. Fisher, "A vibration energy harvesting device with bidirectional resonance frequency tunability," Smart Mater. Struct. 17, 15035 (2008).

${ }^{10}$ R. Naseer, H. L. Dai, A. Abdelkefi, and L. Wang, "Piezomagnetoelastic energy harvesting from vortex-induced vibrations using monostable characteristics," Appl. Energy 203, 142-153 (2017).

${ }^{11}$ W. Liu, C. Liu, X. Li, Q. Zhu, and G. Hu, "Comparative study about the cantilever generators with different curve fixtures,” J. Intell. Mater. Syst. Struct. 29(9), 1884-1899 (2018).

${ }^{12}$ A. Cammarano, S. G. Burrow, D. A. W. Barton, A. Carrella, and L. R. Clare, "Tuning a resonant energy harvester using a generalized electrical load," Smart Mater. Struct. 19, 055003 (2010).

${ }^{13}$ S. P. Beeby et al., "A comparison of power output from linear and nonlinear kinetic energy harvesters using real vibration data," Smart Mater. Struct. 22, 75022 (2013).

${ }^{14} \mathrm{X}$. Y. Li et al., "Piezoelectric material based energy generator using bistable cantilever beam," in Future Wireless Networks and Information Systems (Springer, Berlin, 2012)

${ }^{15}$ B. P. Mann and B. A. Owens, "Investigations of a nonlinear energy harvester with a bistable potential well," J. Sound Vib. 329, 1215-1226 (2010).

${ }^{16}$ R. Masana and M. F. Daqaq, "Energy harvesting in the super-harmonic frequency region of a twin-well oscillator," J. Appl. Phys. 111, 044501 (2012).

${ }^{17}$ I. H. Kim, H. J. Jung, B. M. Lee, and S. J. Jang, "Broadband energy-harvesting using a two degree-of-freedom vibrating body," Appl. Phys. Lett. 98, 214102 (2011).

${ }^{18}$ M. Panyam and M. F. Daqaq, "A comparative performance analysis of electrically optimized nonlinear energy harvesters,” J. Intell. Mater. Syst. Struct. 27, 537-548 (2015).

${ }^{19}$ R. Masana and M. F. Daqaq, "Relative performance of a vibratory energy harvester in mono- and bi-stable potentials," J. Sound Vib. 330, 6036-6052 (2011).

${ }^{20} \mathrm{~J}$. Zha and C. Li, "Synchronization of complex network based on the theory of gravitational field,” Acta Phys. Pol. B 50, 87-114 (2019).

${ }^{21} \mathrm{~J}$. Zha, C. Li, B. Song, and W. Hu, "Synchronisation control of composite chaotic systems,” Int. J. Syst. Sci. 47, 3952-3959 (2016).

${ }^{22}$ Y. Kuramoto and D. Battogtokh, "Coexistence of coherence and incoherence in nonlocally coupled phase oscillators," Nonlinear Phenom. Complex Syst. 4, 380-385 (2002); available at http://www.j-npcs.org/abstracts/vol2002/v5 no4/v5no4p380.html.

${ }^{23}$ D. M. Abrams, L. M. Pecora, and A. E. Motter, "Introduction to focus issue: Patterns of network synchronization," Chaos 26, 094601 (2016).
${ }^{24}$ M. J. Panaggio and D. M. Abrams, "Chimera states: Coexistence of coherence and incoherence in networks of coupled oscillators," Nonlinearity 28, R67 (2015).

${ }^{25}$ D. M. Abrams and S. H. Strogatz, "Chimera states for coupled oscillators," Phys. Rev. Lett. 93, 174102 (2004).

${ }^{26}$ L. V. Gambuzza, A. Buscarino, S. Chessari, L. Fortuna, R. Meucci, and M. Frasca, "Experimental investigation of chimera states with quiescent and synchronous domains in coupled electronic oscillators," Phys. Rev. E 90, 032905 (2014).

${ }^{27}$ E. A. Martens, S. Thutupalli, A. Fourriere, and O. Hallatschek, "Chimera states in mechanical oscillator networks," Proc. Natl. Acad. Sci. U.S.A. 110, 10563-10567 (2013).

${ }^{28}$ L. Larger, B. Penkovsky, and Y. Maistrenko, "Virtual chimera states for delayedfeedback systems," Phys. Rev. Lett. 111, 054103 (2013).

${ }^{29} \mathrm{M}$. Wickramasinghe and I. Z. Kiss, "Spatially organized dynamical states in chemical oscillator networks: Synchronization, dynamical differentiation, and chimera patterns," PLoS One 8, e80586 (2013).

${ }^{30}$ M. R. Tinsley, S. Nkomo, and K. Showalter, "Chimera and phase-cluster states in populations of coupled chemical oscillators," Nat. Phys. 8, 662-665 (2012).

${ }^{31}$ A. M. Hagerstrom, T. E. Murphy, R. Roy, P. Hövel, I. Omelchenko, and E. Schöll, "Experimental observation of chimeras in coupled-map lattices," Nat. Phys. 8, 658-661 (2012).

${ }^{32}$ S. C. Stanton, C. C. McGehee, and B. P. Mann, "Nonlinear dynamics for broadband energy harvesting: Investigation of a bistable piezoelectric inertial generator," Phys. D 239, 640-653 (2010).

${ }^{33}$ W. Wang, J. Cao, C. R. Bowen, D. J. Inman, and J. Lin, "Performance enhancement of nonlinear asymmetric bistable energy harvesting from harmonic, random and human motion excitations," Appl. Phys. Lett. 112, 213903 (2018).

${ }^{34} \mathrm{G}$. Litak and M. Borowiec, "Oscillators with asymmetric single and double well potentials: Transition to chaos revisited," Acta Mech. 184, 47-59 (2006).

${ }^{35}$ A. Wolf, J. B. Swift, H. L. Swinney, and J. A. Vastano, "Determining Lyapunov exponents from a time series," Phys. D: Nonlinear Phenom. 16, 285-317 (1985).

${ }^{36} \mathrm{C}$. Li, X. Wang, and G. Chen, "Diagnosing multistability by offset boosting," Nonlinear Dyn. 90, 1335-1341 (2017).

${ }^{37} \mathrm{C}$. Li, J. C. Sprott, W. Hu, and Y. Xu, "Infinite multistability in a selfreproducing chaotic system," Int. J. Bifurcation Chaos 27, 1750160 (2017).

${ }^{38}$ C. Li, G. Chen, J. Kurths, T. Lei, and Z. Liu, "Dynamic transport: From bifurcation to multistability," Commun. Nonlinear Sci. Numer. Simul. 95, 105600 (2021).

${ }^{39} \mathrm{C}$. Li and J. C. Sprott, "Multistability in the lorenz system: A broken butterfly," Int. J. Bifurcation Chaos 24, 1450131 (2014). 